\title{
$M R$
}

Quantitative assessment of the association between the angiotensin-converting enzyme gene insertion/deletion polymorphism and digestive system cancer risk

\author{
J. Wang*, S. Yang*, F.H. Guo, X. Mao, H. Zhou, Y.Q. Dong, Z.M. Wang and \\ F. Luo \\ Department of General Surgery, HuaShan Hospital, \\ Fudan University School of Medicine, Shanghai, China \\ *These authors contributed equally to this study. \\ Corresponding authors: Z.M. Wang / F. Luo \\ E-mail: wang19701010@126.com / luofen0@126.com
}

Genet. Mol. Res. 14 (4): 14291-14299 (2015)

Received March 25, 2015

Accepted June 17, 2015

Published November 13, 2015

DOI http://dx.doi.org/10.4238/2015.November.13.13

ABSTRACT. The angiotensin-converting enzyme (ACE) gene insertion/ deletion (I/D) polymorphism has been reported to be associated with digestive system cancer; however, the results from previous studies have been conflicting. The present study aimed to investigate the association between the ACE I/D polymorphism and the risk of digestive system cancer using a meta-analysis of previously published studies. Databases were systematically searched to identify relevant studies published prior to December 2014. We estimated the pooled OR with its $95 \% \mathrm{Cl}$ to assess the association. The meta-analysis consisted of thirteen casecontrol studies that included 2557 patients and 4356 healthy controls. Meta-analysis results based on all the studies showed no significant association between the ACE I/D polymorphism and the risk of digestive system cancer (DD vs II: OR $=0.85,95 \% \mathrm{Cl}=0.59-1.24$; $\mathrm{DI}$ vs II: $\mathrm{OR}=$ $0.94,95 \% \mathrm{Cl}=0.78-1.15$; dominant model: $\mathrm{OR}=0.96,95 \% \mathrm{Cl}=0.81-$ 
1.15; recessive model: $\mathrm{OR}=1.06,95 \% \mathrm{Cl}=0.76-1.48)$. Subgroup analyses by race and cancer type did not detect an association between the ACE I/D polymorphism and digestive system cancer risk. However, when the analyses were restricted to smaller studies ( $\mathrm{N}<500$ patients), the summary OR of DI vs II was $0.80(95 \% \mathrm{Cl}=0.66-0.97)$. Our analyses detected a possibility of publication bias with a misestimate of the true association by smaller studies. Overall, meta-analysis results suggest the ACE I/D polymorphism might not be associated with susceptibility to digestive system cancer. Further large and well-designed studies are needed to confirm these conclusions.

Key words: ACE; I/D polymorphism; Digestive system cancer; Genetic polymorphism

\section{INTRODUCTION}

Digestive system cancers are very common worldwide and are major causes of cancerrelated deaths globally (Parkin et al., 2005). These malignancies often exhibit aggressive clinical behavior characterized by local invasiveness and early metastasis. Despite much investigation, the exact mechanism of carcinogenesis is not yet fully elucidated. Inflammation, smoking, alcohol, and low-nutrition intake are known to be the main exogenous contributors to the risk of digestive system cancer (Zhao et al., 2014). Although these factors have been documented to influence the risk of digestive system cancer, not all individuals with these features develop the disease, even though they are exposed in the same environment as those that do. This indicates that other factors might contribute to digestive system cancer pathogenesis. With the developments in molecular biology, researchers have provided strong evidence that genetic factors are important in the pathogenesis of cancer (Thompson et al., 2009; Fujimori et al., 2012).

The renin-angiotensin system (RAS) has been implicated in the regulation of blood pressure and cardiovascular homeostasis (van der Knapp et al., 2008). Angiotensin II, the main biologically active peptide of the RAS, is converted from angiotensin I via the angiotensin-converting enzyme (ACE). ACE is differentially expressed in several carcinomas and might affect tumor cell proliferation, migration, and angiogenesis (Röcken et al., 2005). The ACE gene is located on 17q23 in humans (Jeunemaitre et al., 1992). There is a common insertion/deletion (I/D) polymorphism in this gene characterized by the presence or absence of a 287-bp Alu repetitive sequence in intron 16 (Castellon and Hamdi, 2007). Homozygotes for the D allele have the highest ACE plasma levels, homozygotes for the I allele have the lowest, and ID heterozygotes have intermediate levels (Rigat et al., 1990).

To date, many studies have shown that the ACE I/D polymorphism might be closely associated with the risk of cancers, including lung and breast cancer (Cheon et al., 2000; Namazi et al., 2010). In addition, several studies have found an association between the ACE I/D polymorphism and digestive system cancer risk. However, the published results remain controversial, possibly due to small sample sizes, low statistical power, and clinical heterogeneity (Ammar et al., 2012). In the present study, therefore, we performed a meta-analysis to examine whether the ACE I/D polymorphism was associated with the risk of digestive system cancer based on all eligible published case-control studies. 


\section{MATERIAL AND METHODS}

\section{Study selection}

A computerized literature search was carried out in the US National Library of Medicine PubMed database, along with ISI Web of Knowledge, Medline, Embase, and Google Scholar Search databases (updated to December 2014) by two investigators independently to collect articles on case-control studies examining the association of the ACE I/D polymorphism with digestive system cancer. References of the retrieved articles were also screened for original studies (J.W. and S.Y.). The following search terms were used: "ACE", "digestive system cancer", "polymorphism", "single nucleotide polymorphism", and "genetic polymorphism". The reference lists of major textbooks, reviews, and included articles were identified through manual searches to find other potentially eligible studies.

\section{Inclusion and exclusion criteria}

Studies included in this meta-analysis met the following criteria: 1) case-control studies that addressed patients with digestive system cancer and healthy controls; 2) studies on the association of the ACE I/D polymorphism and susceptibility to digestive system cancer; and 3) studies that included sufficient genotype data for extraction. Studies were excluded if they were: 1) not a case-control study; 2) duplicates of previous publications; 3) based on incomplete data; or 4) meta-analyses, case reports, letters, reviews, or editorial articles.

\section{Quality assessment}

The quality of these studies was also evaluated independently by the same two investigators according to the predefined quality assessment rules shown in Table 1 (Jiang et al., 2012). The criteria covered the representativeness of cases, source of controls, ascertainment of digestive system cancer, total sample size, quality control of genotyping methods, and Hardy-Weinberg equilibrium (HWE) in the control population. Disagreements were resolved by consensus. The total score ranged from 0 (worst) to 15 (best). Papers scoring <10 were classified as "low quality" and those scoring $\geq 10$ as "high quality".

\section{Data extraction}

Information was extracted from all eligible publications independently by 2 investigators according to the inclusion criteria listed above. Disagreements were settled by discussion among all reviewers. The following characteristics were collected from each study: first author, year of publication, country, nationality, numbers of patients and controls, gene polymorphisms, and evidence of HWE.

\section{Statistical analysis}

The Fisher exact test (http://ihg.gsf.de/cgi-bin/hw/hwa1.pl) was used to test whether the distributions of genotypes among controls was in accord with HWE. The strength of the associations 
between the $A C E \mathrm{I} / \mathrm{D}$ polymorphism and the susceptibility to digestive system cancer were estimated by $\mathrm{OR}$ and $95 \% \mathrm{Cl}$ under homozygote comparison (DD vs II), heterozygote comparison (DI vs II), dominant (DD+DI vs II), and recessive (II+DI vs DD) models between groups. Heterogeneity was tested by using a $Q$-test. A fixed effect model was used to pool the data when the $P$ value of the $Q$-test was $\geq 0.05$; otherwise, a random effect model was selected. We also performed sensitivity and subgroup analyses to explore the reason of heterogeneity. Subgroup analysis was performed to test the effect of cancer type, country, and sample size. Potential publication bias was evaluated using the funnel plot and the Begg test. Statistical analysis was conducted using the STATA statistical package version 12.0 (STATA, College Station, TX, USA).

\begin{tabular}{ll} 
Table 1. Scale for quality assessment. & \\
\hline Criteria & Score \\
\hline Source of patients & 3 \\
Selected from population or cancer registry & 2 \\
Selected from hospital & 1 \\
Selected from pathology archives, but without description & 0 \\
Not described & 3 \\
Source of controls & 2 \\
Population-based & 1 \\
Blood donors or volunteers & 0 \\
Hospital-based (cancer-free patients) & \\
Not described & 3 \\
Specimens from patients used to determine genotypes & 0 \\
White blood cells or normal tissues & \\
Tumor tissues or exfoliated cells of tissue & 3 \\
Hardy-Weinberg equilibrium in controls & 0 \\
Hardy-Weinberg equilibrium & \\
Hardy-Weinberg disequilibrium & 3 \\
Total sample size & 3 \\
$\geq 1000$ & 2 \\
$\geq 500$ but <1000 & 3 \\
$\geq 200$ but $<500$ & 0 \\
$>0$ but <200 & 0 \\
\hline
\end{tabular}

\section{RESULTS}

\section{Characteristics of included studies}

The literature search identified a total of 71 potentially relevant papers. Of these, 54 were excluded because of obvious irrelevance by reading the titles and abstracts, leaving 17 articles for full publication review. Of these, 4 were excluded for a variety of reasons. Finally, a total of 13 studies were included in our meta-analysis, which included 2557 patients with cancer and 4356 controls (Goto et al., 2005; Sugimoto et al., 2006; Nikiteas et al., 2007; Röcken et al., 2005, 2007; Toma et al., 2009; Vairaktaris et al., 2009; Hibi et al., 2011; Kupcinskas et al., 2011; Liu et al., 2011, 2012; Yuan et al., 2013; Zha et al., 2014). A flow diagram of the study identification and selection is shown in Figure 1. All the studies were published between 2005 and 2014. The genotype distributions among the controls of all studies were consistent with HWE. All included studies were of high quality as the quality score assessment of each one was higher than or equal to 10 points. All the articles were written in English. There were 7 studies of Asians and 6 of Caucasians. The types of cancers in these studies included gastric cancer $(N=5)$, colorectal cancer $(N=4)$, oral cancer $(\mathrm{N}=2)$, and hepatocellular carcinoma ( $\mathrm{HCC}, \mathrm{N}=2)$. The main characteristics of the studies included are summarized in Table 2. 


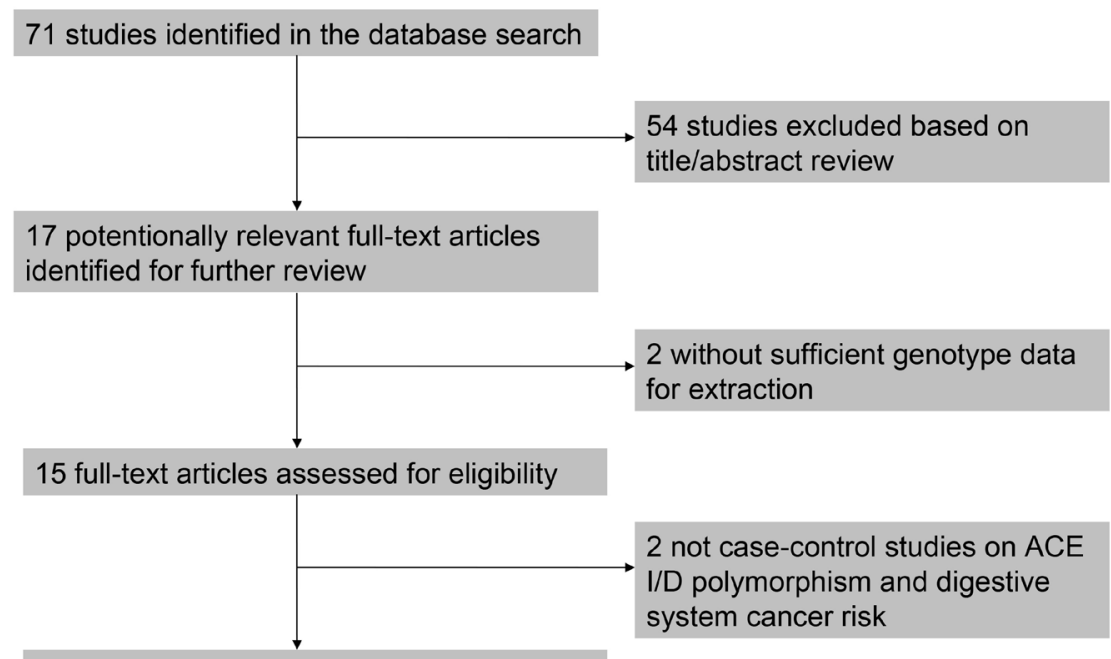

13 studies included in the meta-analysis

Figure 1. Flow diagram of study identification. $A C E \mathrm{I} / \mathrm{D}=$ angiotensin-converting enzyme gene insertion/deletion (polymorphism).

\begin{tabular}{|c|c|c|c|c|c|c|c|c|c|c|c|c|}
\hline \multirow[t]{2}{*}{ Study included } & \multirow[t]{2}{*}{ Area } & \multirow[t]{2}{*}{ Race } & \multirow[t]{2}{*}{ Cancer type } & \multirow{2}{*}{$\begin{array}{l}\text { Patients/ } \\
\text { Controls }\end{array}$} & \multicolumn{3}{|c|}{ Genotypes for patients } & \multicolumn{3}{|c|}{ Genotypes for controls } & \multirow{2}{*}{$\begin{array}{c}\text { HWE } \\
\text { test }\end{array}$} & \multirow{2}{*}{$\begin{array}{l}\text { Quality } \\
\text { scores }\end{array}$} \\
\hline & & & & & ॥ & ID & $\mathrm{DD}$ & II & ID & $\mathrm{DD}$ & & \\
\hline Goto (2005) & Japan & Asian & Gastric & $202 / 454$ & 76 & 98 & 28 & 209 & 189 & 56 & 0.20 & 13 \\
\hline Rocken (2005) & Germany & Caucasian & Gastric & $113 / 189$ & 24 & 57 & 32 & 41 & 95 & 53 & 0.90 & 10 \\
\hline Sugimoto (2006) & Japan & Asian & Gastric & $119 / 132$ & 54 & 53 & 12 & 50 & 60 & 22 & 0.58 & 10 \\
\hline Nikiteas (2007) & Greece & Caucasian & Colorectal & $92 / 102$ & 15 & 27 & 50 & 6 & 44 & 52 & 0.40 & 10 \\
\hline Rocken (2007) & Germany & Caucasian & Colorectal & $141 / 189$ & 37 & 69 & 35 & 41 & 95 & 53 & 0.90 & 10 \\
\hline Toma (2009) & Romania & Caucasian & Colorectal & $108 / 150$ & 25 & 50 & 33 & 30 & 73 & 47 & 0.86 & 10 \\
\hline Vairaktaris (2009) & Greece & Caucasian & Oral & $160 / 153$ & 30 & 70 & 60 & 9 & 66 & 78 & 0.30 & 11 \\
\hline Liu (2011) & China & Asian & Colorectal & $241 / 299$ & 71 & 138 & 32 & 95 & 158 & 46 & 0.14 & 14 \\
\hline Hibi (2011) & Japan & Asian & Gastric & $582 / 1740$ & 252 & 255 & 75 & 745 & 791 & 204 & 0.79 & 13 \\
\hline Kupcinskas (2011) & Germany & Caucasian & Gastric & $114 / 238$ & 27 & 59 & 28 & 62 & 110 & 66 & 0.24 & 11 \\
\hline Liu (2012) & China & Asian & Oral & $186 / 120$ & 78 & 50 & 58 & 57 & 54 & 9 & 0.43 & 13 \\
\hline Yuan (2013) & China & Asian & $\mathrm{HCC}$ & $289 / 384$ & 59 & 214 & 16 & 84 & 211 & 89 & 0.05 & 11 \\
\hline Zha (2014) & China & Asian & $\mathrm{HCC}$ & $210 / 206$ & 50 & 103 & 57 & 57 & 115 & 34 & 0.06 & 12 \\
\hline
\end{tabular}

HWE = Hardy-Weinberg equilibrium; I = insertion; $\mathrm{D}=$ deletion; HCC = hepatocellular carcinoma.

\section{Quantitative synthesis}

A summary of the meta-analysis findings of the association between the ACE I/D polymorphism and digestive system cancer risk is shown in Figure 2 and Table 3. The combined results based on all studies showed that the $A C E \mathrm{I} / \mathrm{D}$ polymorphism was not associated with digestive system cancer risk in the population as a whole $(\mathrm{DD}$ vs II: $\mathrm{OR}=0.85,95 \% \mathrm{Cl}=0.59-1.24$; $\mathrm{DI}$ vs II: $\mathrm{OR}=0.94,95 \% \mathrm{Cl}=0.78-1.15$; dominant model: $\mathrm{OR}=0.96,95 \% \mathrm{Cl}=0.81-1.15$; recessive model: $\mathrm{OR}=1.06,95 \% \mathrm{Cl}=0.76-1.48)$. Sensitivity analysis was performed by successively deleting one single study from the overall pooled analysis to check the influence of the removed data. However, the results revealed that no single study changed the between-study heterogeneities (Figure 3). 


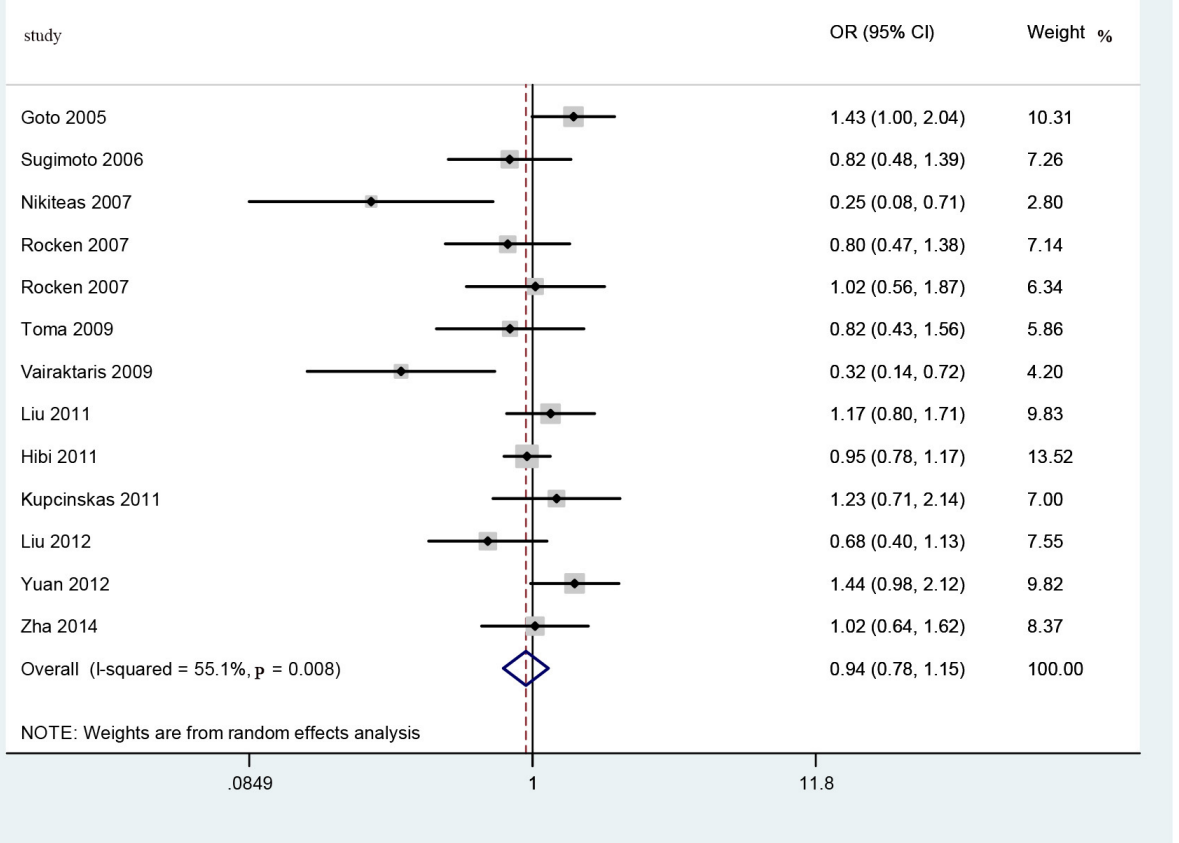

Figure 2. Meta-analysis of the association between the ACE I/D polymorphism and digestive system cancer risk (DI vs II). ACE $\mathrm{I} / \mathrm{D}=$ angiotensin-converting enzyme gene insertion/deletion.

\begin{tabular}{|c|c|c|c|c|c|c|c|c|c|}
\hline \multirow[t]{2}{*}{ Variable } & \multirow[t]{2}{*}{$\mathrm{N}$} & \multicolumn{2}{|c|}{ Homozygous co-dominant } & \multicolumn{2}{|c|}{ Heterozygous co-dominant } & \multicolumn{2}{|c|}{ Dominant model } & \multicolumn{2}{|c|}{ Recessive model } \\
\hline & & DD vs II & $\overline{\mathrm{P}_{\text {het }}{ }^{a}}$ & DI vs II & $\overline{P_{h e t}{ }^{a}}$ & $\mathrm{DD}+\mathrm{DI}$ vs II & $\overline{\mathrm{P}_{\text {het }} a}$ & II+DI vs DD & $\mathrm{P}_{\text {het }}{ }^{a}$ \\
\hline All & 13 & $0.85(0.59-1.24)$ & 0.000 & $0.94(0.78-1.15)$ & 0.008 & $0.96(0.81-1.15)$ & 0.015 & $1.06(0.76-1.48)$ & 0.000 \\
\hline \multicolumn{10}{|l|}{ Ethnicity } \\
\hline Asian & 7 & $1.07(0.62-1.85)$ & 0.000 & $1.02(0.79-1.32)$ & 0.011 & $1.08(0.95-1.22)$ & 0.378 & $0.98(0.53-1.81)$ & 0.000 \\
\hline Caucasian & 6 & $0.66(0.42-1.02)$ & 0.050 & $0.71(0.46-1.10)$ & 0.027 & $0.70(0.46-1.06)$ & 0.023 & $1.17(0.95-1.44)$ & 0.471 \\
\hline \multicolumn{10}{|l|}{ Cancer type } \\
\hline Gastric & 5 & $1.05(0.84-1.30)$ & 0.364 & $0.96(0.68-1.35)$ & 0.019 & $1.04(0.90-1.20)$ & 0.242 & $0.99(0.81-1.21)$ & 0.477 \\
\hline Colorectal & 4 & $0.77(0.55-1.07)$ & 0.508 & $0.78(0.48-1.26)$ & 0.051 & $0.88(0.68-1.13)$ & 0.121 & $1.08(0.83-1.39)$ & 0.842 \\
\hline Oral & 2 & $1.05(0.05-20.10)$ & 0.000 & $0.50(0.24-1.03)$ & 0.125 & $0.60(0.13-2.71)$ & 0.001 & $0.57(0.06-5.40)$ & 0.000 \\
\hline $\mathrm{HHC}$ & 2 & $0.70(0.09-5.05)$ & 0.000 & $1.26(0.93-1.69)$ & 0.259 & $1.15(0.86-1.52)$ & 0.834 & $1.65(0.18-15.50)$ & 0.000 \\
\hline \multicolumn{10}{|l|}{ Sample size } \\
\hline$\geq 500$ & 4 & $0.80(0.43-1.47)$ & 0.000 & $1.12(0.97-1.29)$ & 0.114 & $1.08(0.94-1.24)$ & 0.326 & $1.28(0.95-1.56)$ & 0.000 \\
\hline$<500$ & 9 & $0.88(0.52-1.50)$ & 0.000 & $0.80(0.66-0.97)$ & 0.078 & $0.83(0.62-1.11)$ & 0.015 & $0.91(0.62-1.35)$ & 0.000 \\
\hline
\end{tabular}

$\mathrm{HHC}=$ hepatocellular carcinoma; ${ }^{\mathrm{P}}$ value of the $\mathrm{Q}$-test for heterogeneity.

\section{Subgroup analysis}

Considering the potential impact of confounding factors on the overall results, we further performed subgroup analyses. When stratified according to ethnicity, the ACE I/D polymorphism was not found to be associated with digestive system cancer in Asians or Caucasians (Table 
2). When stratified according to cancer type, the ACE I/D polymorphism was not found to be associated with gastric cancer, colorectal cancer, oral cancer, or HCC. Further subgroup analysis using sample size also detected no significant association with sample size $\geq 500$. However, when limiting the analysis to the study sample size $<500$, we detected significant association between ACE I/D polymorphism and digestive system cancer (DI vs II: $\mathrm{OR}=0.80,95 \% \mathrm{Cl}=0.66-0.97$ ).

\section{Publication bias}

A funnel plot and the Begg test were used to assess the publication bias of the sampled studies. The results demonstrated no evidence of publication bias in our study, suggesting that the publication bias was low in the present meta-analysis (all $\mathrm{P}>0.05$ ).

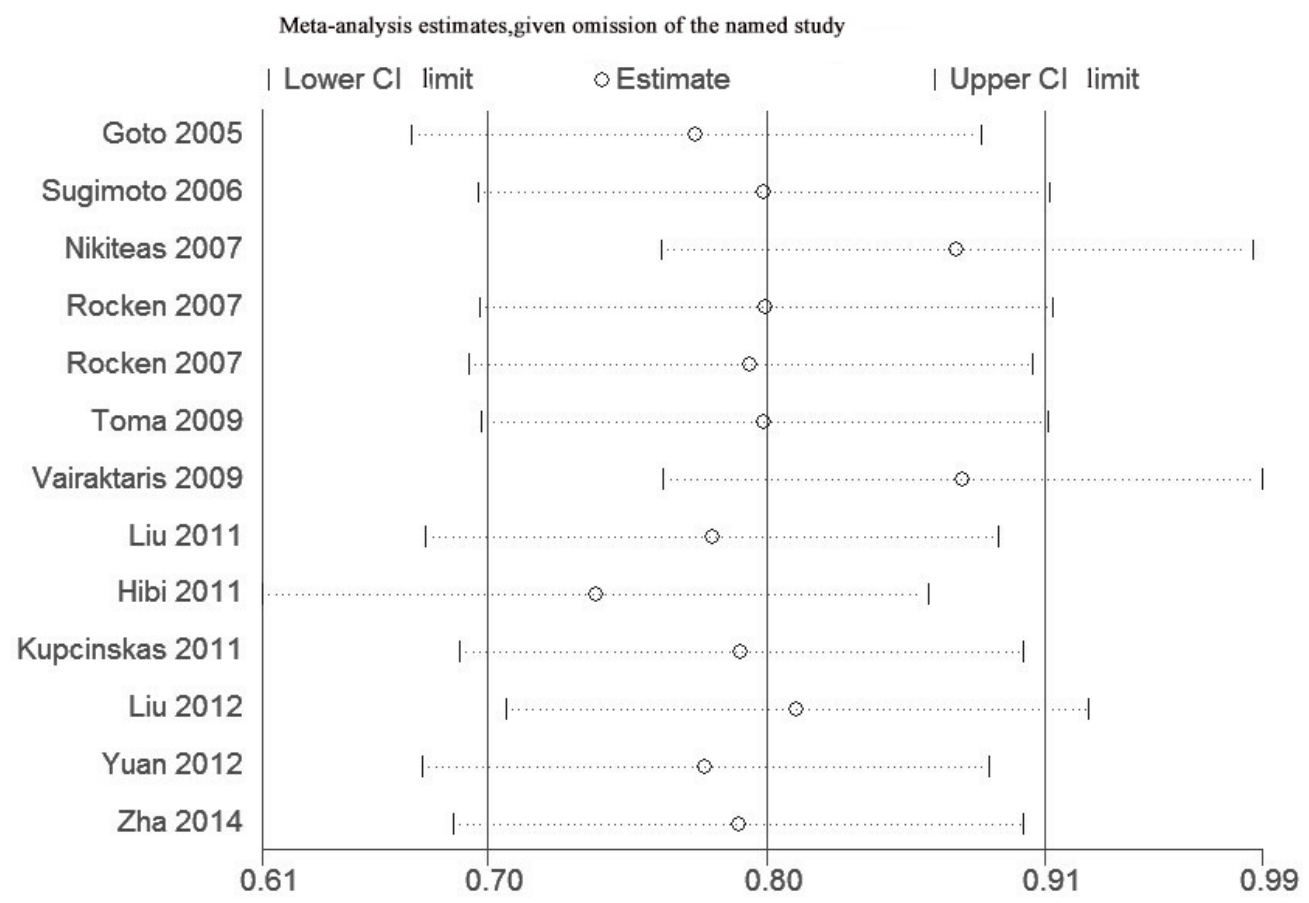

Figure 3. One-way sensitivity analysis of the pooled ORs and $95 \% \mathrm{Cls}$ for the $A C E$ I/D polymorphism, from sequential omission of each dataset in the meta-analysis (DI vs II). ACE I/D = angiotensin-converting enzyme gene insertion/deletion.

\section{DISCUSSION}

The ACE I/D polymorphism has been found to influence serum ACE activity, and DD carriers have increased ACE levels (Rigat et al., 1990). Some studies have shown that the ACE I/D polymorphism was associated with several cancer types including lung and breast cancer by influencing tumor cell proliferation, tumor cell migration, and angiogenesis (Haiman et al., 2003). However, to our knowledge, the relationship between the ACE I/D polymorphism and the risk of digestive system cancer has not been completely clarified. The most likely reason for the 
inconsistencies among these studies is that they are single case-control studies with small sample sizes. Therefore, we conducted this meta-analysis to combine the same type of studies to increase the sample size and statistical power, and thereby obtain a more authentic result.

The present meta-analysis, including 2557 patients and 4356 controls from 13 casecontrol studies, explored the association between the ACE I/D polymorphism and digestive system cancer risk. The results of the present meta-analysis revealed that the ACE I/D polymorphism is not associated with increased or decreased risk of digestive system cancer in the overall population. Because of the difference in genetic backgrounds and the environments in which the subjects lived, we performed an ethnicity-specific subgroup analysis, and detected no significant association among Asians or Caucasians, in line with the overall data. Similarly, in the subgroup analysis based on cancer type, significantly increased and decreased cancer risks were not observed for gastric cancer, colorectal cancer, oral cancer, or HCC. However, as the present meta-analysis also involved several studies with a small sample size, there might have been a selective bias for the association between the ACE I/D polymorphism and digestive system cancer development. Accordingly, following stratification by sample size $(<500)$, this meta-analysis detected significant association, suggesting a possibility of publication bias in the smaller studies. Therefore, caution should be exercised when considering this conclusion.

The present study has some limitations. First, in this meta-analysis, the primary articles only provided data on Caucasians and Asians. Data regarding other ethnicities, such as Africans, were not available. Second, subgroup analyses on age, gender, smoking, alcohol, and other factors (such as Helicobacter pylori infection, an important risk factor for digestive system cancer) were not conducted in the present study due to insufficient relevant data available in the primary studies. Third, the sample sizes for a proportion of studies included were relatively small; the matching criteria for the cases and controls were also not strict. Thus, bias might exist. Further investigations with larger sample sizes and strict matching criteria are needed to address the possible associations. Finally, as only published English studies were included in this study, publication and potential language biases might have occurred.

In conclusion, our meta-analysis indicates that the ACE I/D polymorphism is not associated with risk of digestive system cancer. Owing to the above-mentioned limitations, the findings should be verified by further research in the near future.

\section{Conflicts of interest}

The authors declare no conflict of interest.

\section{REFERENCES}

Ammar M, Bouazizi F, Bouhaha R, Zaraa J, et al. (2012). Association analysis of LCE3C-LCE3B deletion in Tunisian psoriatic population. Arch. Dermatol. Res. 304: 733-738.

Castellon R and Hamdi HK (2007). Demystifying the ACE polymorphism: from genetics to biology. Curr. Pharm. Des. 13: 1191-1198.

Cheon KT, Choi KH, Lee HB, Park SK, et al. (2000). Gene polymorphisms of endothelial nitric oxide synthase and angiotensin converting enzyme in patients with lung cancer. Lung 178: 351-360.

Fujimori Y, Inokuchi M, Takagi Y, Kato K, et al. (2012). Prognostic value of RKIP and p-ERK in gastric cancer. J. Exp. Clin. Cancer Res. 31: 30.

Goto Y, Ando T, Nishio K, Ishida Y, et al. (2005). The ACE gene polymorphism is associated with the incidence of gastric cancer among H. pylori seropositive subjects with atrophic gastritis. Asian Pac. J. Cancer Prev. 6: 464-467. 
Haiman CA, Henderson SO, Bretsky P, Kolonel LN, et al. (2003). Genetic variation in angiotensin I-converting enzyme (ACE) and breast cancer risk: The multiethnic cohort. Cancer Res. 63: 6984-6987.

Hibi S, Goto Y, Ando T, Matsuo K, et al. (2011). No association between angiotensin I converting enzyme (ACE) I/D polymorphism and gastric cancer risk among Japanese. Nagoya J. Med. Sci. 73: 169-175.

Jeunemaitre X, Lifton RP, Hunt SC, Williams RR, et al. (1992). Absence of linkage between the angiotensin converting enzyme locus and human essential hypertension. Nat. Genet. 1: 72-75.

Jiang DK, Ren WH, Yao L, Wang WZ, et al. (2012). Meta-analysis of association between TP53 Arg72Pro polymorphism and bladder cancer risk. Urology 76: 765.e1-7.

Kupcinskas J, Wex T, Bornschein J, Selgrad M, et al. (2011). Lack of association between gene polymorphisms of angiotensin converting enzyme, nod-like receptor 1, toll-like receptor 4, FAS/FASL and the presence of Helicobacter pylori-induced premalignant gastric lesions and gastric cancer in Caucasians. BMC Med. Genet. 12: 112.

Liu SY, Sima X, Wang CH and Gao M (2011). The association between ACE polymorphism and risk of colorectal cancer in a Chinese population. Clin. Biochem. 44: 1223-1226.

Liu YT, Lin LW, Chen CY, Wang CP, et al. (2012). Polymorphism of angiotensin I-converting enzyme gene is related to oral cancer and lymph node metastasis in male betel quid chewers. Oral Oncol. 48: 1257-1262.

Namazi S, Monabati A, Ardeshir-Rouhani-Fard S and Azarpira N (2010). Association of angiotensin I converting enzyme (insertion/deletion) and angiotensin II type 1 receptor (A1166C) polymorphisms with breast cancer prognostic factors in Iranian population. Mol. Carcinog. 49: 1022-1030.

Nikiteas N, Tsigris C, Chatzitheofylaktou A and Yannopoulos A (2007). No association with risk for colorectal cancer of the insertion/deletion polymorphism which affects levels of angiotensin-converting enzyme. In Vivo 21: 1065-1068.

Parkin DM, Bray F, Ferlay J and Pisani P (2005). Global cancer statistics 2002. CA Cancer J. Clin. 55: 74-108.

Rigat B, Hubert C, Alhenc-Gelas F, Cambien F, et al. (1990). An insertion/deletion polymorphism in the angiotensin I converting enzyme gene accounting for half the variance of serum enzyme levels. J. Clin. Invest. 86: 1343-1346.

Röcken C, Lendeckel U, Dierkes J, Westphal S, et al. (2005). The number of lymph node metastases in gastric cancer correlates with the angiotensin I-converting enzyme gene insertion/deletion polymorphism. Clin. Cancer Res. 11: 2526-2530.

Röcken C, Neumann K, Carl-McGrath S, Lage H, et al. (2007). The gene polymorphism of the angiotensin I-converting enzyme correlates with tumor size and patient survival in colorectal cancer patients. Neoplasia 9: 716-722.

Sugimoto M, Furuta T, Shirai N, Ikuma M, et al. (2006). Influences of chymase and angiotensin I-converting enzyme gene polymorphisms on gastric cancer risks in Japan. Cancer Epidemiol. Biomarkers Prev. 15: 1929-1934.

Thompson CL, PLummer SJ, Merkulova A, Cheng I, et al. (2009). No association between cyclooxygenase-2 and uridine diphosphate glucuronosyltransferase $1 \mathrm{~A} 6$ genetic polymorphisms and colon cancer risk. World J. Gastroenterol. 15: 2240-2244.

Toma M, Cimponeriu D, Apostol P, Stavarachi M, et al. (2009). Lack of association between ACE ID polymorphism and colorectal cancer in Romanian patients. Chirurgia 104: 553-556.

Vairaktaris E, Serefoglou Z, Avgoustidis D, Yapijakis C, et al. (2009). Gene polymorphisms related to angiogenesis, inflammation and thrombosis that influence risk for oral cancer. Oral Oncol. 45: 247-253.

van der Knaap R, Siemens C, Coebergh JW, van Duijn CM, et al. (2008). Reninangiotensin system inhibitors, angiotensin I-converting enzyme gene insertion/deletion polymorphism and cancer: the Rotterdam Study. Cancer 112: 748-757.

Yuan F, Zhang LS, Li HY, Liao M, et al. (2013). Influence of angiotensin I-converting enzyme gene polymorphism on hepatocellular carcinoma risk in China. DNA Cell Biol. 32: 268-273.

Zha Y, Gan P, Liu Q and Tan J (2014). Relationship between polymorphism of angiotensin-converting enzyme gene insertion/ deletion and risk of hepatocellular carcinoma in a Chinese Dai population. J. Renin Angiotensin Aldosterone Syst. [Epub Ahead of Print].

Zhao F, Cao Y, Zhu H, Huang M, et al. (2014). The $-765 \mathrm{G}>\mathrm{C}$ polymorphism in the cyclooxygenase-2 gene and digestive system cancer: a meta-analysis. Asian Pac. J. Cancer Prev. 15: 8301-8310. 